\title{
Parametric Studies of Square Solar Sails Using Finite Element Analysis
}

\author{
David W. Sleight ${ }^{*}$ and Danniella M. Muheim ${ }^{\dagger}$ \\ NASA Langley Research Center, Hampton, VA 23681-2199, U.S.A.
}

\begin{abstract}
Parametric studies are performed on two generic square solar sail designs to identify parameters of interest. The studies are performed on systems-level models of full-scale solar sails, and include geometric nonlinearity and inertia relief, and use a Newton-Raphson scheme to apply sail pre-tensioning and solar pressure. Computational strategies and difficulties encountered during the analyses are also addressed. The purpose of this paper is not to compare the benefits of one sail design over the other. Instead, the results of the parametric studies may be used to identify general response trends, and areas of potential nonlinear structural interactions for future studies. The effects of sail size, sail membrane pre-stress, sail membrane thickness, and boom stiffness on the sail membrane and boom deformations, boom loads, and vibration frequencies are studied. Over the range of parameters studied, the maximum sail deflection and boom deformations are a nonlinear function of the sail properties. In general, the vibration frequencies and modes are closely spaced. For some vibration mode shapes, local deformation patterns that dominate the response are identified. These localized patterns are attributed to the presence of negative stresses in the sail membrane that are artifacts of the assumption of ignoring the effects of wrinkling in the modeling process, and are not believed to be physically meaningful. Over the range of parameters studied, several regions of potential nonlinear modal interaction are identified.
\end{abstract}

\section{Introduction}

Solar sail concepts are of interest for future space exploration missions ${ }^{1}$. Solar sails span very large areas to capture and reflect photons from the Sun and are propelled through space by momentum gained from the photons $^{2,3,4}$. (See Fig. 1). Because solar sails utilize a cost effective source of propellantless propulsion, they offer potential advantages over traditional, chemical propulsion spacecraft. A square solar sail will be used by the Team Encounter mission, and is proposed for the National Oceanic and Atmospheric Administration Geostorm Warning mission ${ }^{4,6,7}$. The Team Encounter solar sail is $76 \mathrm{~m}$ per edge, while the Geostorm sail will be $70 \mathrm{~m}$ per edge. Future solar sail missions include inner and outer planetary and interstellar missions with sails up to $1,000 \mathrm{~m}$ per edge $\mathrm{e}^{2}$. The world's largest thermal vacuum chamber is located at the NASA Glenn Research Center, Plum Brook Station, and is $100 \mathrm{ft}$. in diameter and $122 \mathrm{ft}$. high. Clearly, this facility is not large enough to perform ground tests on full-scaled solar sails. In addition, the influence of gravity makes full-scale structural response measurements via ground tests difficult and unrepresentative of the space environment. Therefore, computational methods, models and tools to predict solar sail structural response must be developed, validated, and verified by both test-analysis correlation with sub-scale models and systems-level studies.

Solar sails with ultra-thin membranes and long slender booms present

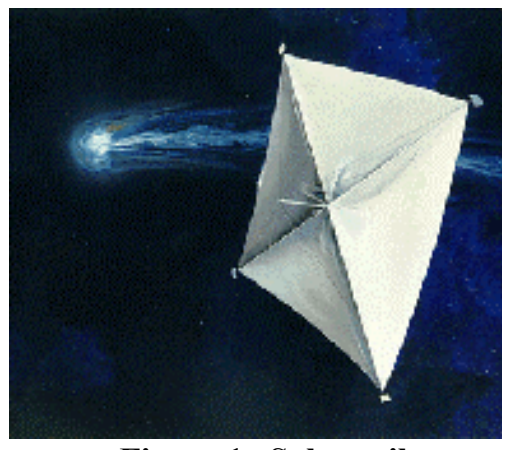

Figure 1: Solar sail. analysis challenges because of their extreme dimensions and geometrically nonlinear behavior. An earlier assessment of a square solar sail using finite element analysis (FEA) with beam, bar and membrane elements was recently published ${ }^{8}$. Other efforts to predict solar sail structural response include design and closed-formed analytical studies ${ }^{7,9}$ of solar sails up to $400 \mathrm{~m}$ per edge. Greschik and Mikulas ${ }^{9}$ evaluated several square solar sails

\footnotetext{
* Aerospace Engineer, Analytical and Computational Methods Branch/Structures and Materials Competency, MS 240, AIAA Member.

$\dagger$ Aerospace Engineer, Analytical and Computational Methods Branch/Structures and Materials Competency, MS 240, AIAA Senior Member.
} 
with different sail-to-boom connection systems. Analytical studies using FEA have been performed by Murphy et al. ${ }^{10}$ for sail sizes up to $300 \mathrm{~m}$ per edge with membrane elements and by Taleghani et al. ${ }^{11}$ for component and system-level solar sails models up to $150 \mathrm{~m}$ per edge with shell elements. The work in Ref. 11 focused on methods and models by comparing the results from two commercial FEA programs.

The objectives of this paper are two-fold: to perform parametric studies on generic solar sail designs to identify parameters of interest for future research efforts and to perform system-level analyses of full-scale solar sails. When computational difficulties are encountered, the strategies used to mitigate them are also described. The work reported in Ref. 11 is extended to include parametric studies of system-level solar sail models using ABAQUS v6.4$1^{12}$. The studies described in this paper may be used to identify the general trends in the structural responses associated with key parameters in the design of solar sails, and areas of potential nonlinear structural interactions for future studies.

\section{Solar Sail Model Descriptions}

Two of the sail design concepts studied in Ref. 9 will be more closely examined herein. One concept has four separate triangular sail regions attached to the booms by cables at five points as shown in Fig. 2a) and is referred to as the five-point connected sail. The other concept has four separate triangular sail regions attached to the booms by multiple cables along the length of the booms as shown in Fig. 2b) and is referred to as the multiple-point connected sail. The multiple cables approximate a continuously connected sail and boom configuration.

In this study, both solar sail design

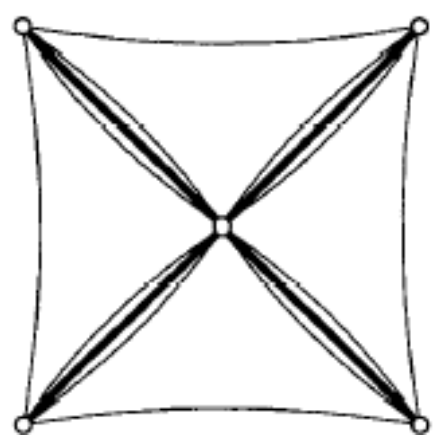

a) Five-point connected sail

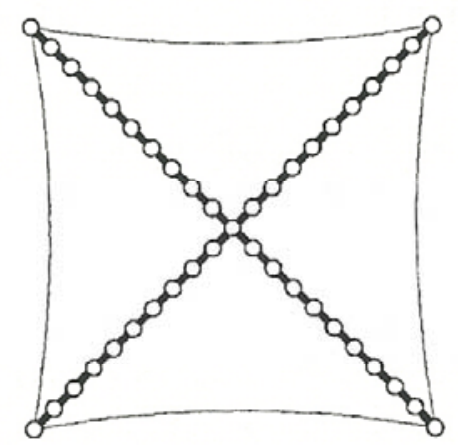

b) Multiple-point connected sail

Figure 2: Square solar sail design concepts'. concepts are adapted from the $150 \mathrm{~m}$ per edge solar sail in Ref. 7, hereby referred to as the $150 \mathrm{~m}$ solar sail. Common to both concepts are four $106 \mathrm{~m}$ booms, four triangular sail membrane regions, a $2 \mathrm{~m}$ control mast, concentrated masses, and cables that connect the sail membrane quadrants to the boom. A concentrated mass representing a $0.58 \mathrm{~kg}$ tip plate is located at the end of each boom and a concentrated mass representing a $228 \mathrm{~kg}$ science payload mass is located at the end of the control mast. In addition, a concentrated mass of $54.84 \mathrm{~kg}$ is located at the central spacecraft bus, which includes mass contributions from control wheels, gimbals, fittings, parts, instrumentation, and thermal control devices.

The $106 \mathrm{~m}$ booms are idealized as thin-walled tubes, each with a circular cross-section radius of $0.229 \mathrm{~m}$ and a baseline thickness of $7.5 \mu \mathrm{m}$. The control mast is idealized as a thin-walled tube with a circular cross-section and isotropic material properties in the finite element model. The control mast is sized so that its lowest natural frequency including the $228 \mathrm{~kg}$ science payload mass is $0.5 \mathrm{~Hz}$ based on the closed-form frequency of a lumped mass at one end of a column with the other end fixed. The chosen control mast sizing avoids interaction with the fundamental modes of the sail system based on the previous study ${ }^{11}$. The cables connecting the sail membrane to the booms are idealized as a thin-walled tube with isotropic material properties with nominal stiffness similar to Kevlar $^{\mathrm{TM}}$, and have a solid circular cross-section with a radius of $0.78 \mu \mathrm{m}$. The solar sail membrane is assigned isotropic material properties similar to Kapton ${ }^{\mathrm{TM}}$. The baseline thickness of the sail membrane is $2.50 \mu \mathrm{m}$. An effective mass density of $1572 \mathrm{~kg} / \mathrm{m}^{3}$ includes the additional mass of metallic coatings, and reinforcement seams. The solar sail properties are included in Table 1.

Table 1: Baseline $150 \mathrm{~m}$ solar sail properties.

\begin{tabular}{ccccccc}
\hline \hline Components & Material & $\begin{array}{c}\text { Radius } \\
(\mathbf{m})\end{array}$ & $\begin{array}{c}\text { Thickness } \\
(\mathbf{m})\end{array}$ & $\begin{array}{c}\text { Modulus } \\
\left(\mathbf{N} / \mathbf{m}^{2}\right)\end{array}$ & $\begin{array}{c}\text { Poisson's } \\
\text { Ratio }\end{array}$ & $\begin{array}{c}\text { Density } \\
\left(\mathbf{k g} / \mathbf{m}^{3}\right)\end{array}$ \\
\hline Booms & Composite & 0.229 & $7.5 \times 10^{-6}$ & $124 \times 10^{9}$ & 0.30 & 1908 \\
Cables & Kevlar & 0.0005 & N/A & $62 \times 10^{9}$ & 0.36 & 1440 \\
Control mast & Isotropic & 0.01705 & 0.005 & $124 \times 10^{9}$ & 0.30 & 7660 \\
Membrane & Kapton & N/A & $2.50 \times 10^{-6}$ & $2.48 \times 10^{9}$ & 0.34 & 1572 \\
\hline \hline
\end{tabular}


Finite element models of the five-point and multiple-point connected sails are shown in Figs. 3 and 4 , respectively. All finite element models were analyzed with the ABAQUS v6.4.1 finite element code. The solar sail booms and control mast were modeled with many two-node B31 beam elements, whereas each cable connecting the sail membranes to the boom was modeled as a single two-node T3D2 truss element. The four triangular sail membrane regions were modeled with three-node triangular M3D3 membrane elements. The finite element model of the multiple-point connected solar sail was adapted from the five-point connected solar sail model by adding cables every 1.6 meters along the length of the booms to connect the triangular sail regions to the booms. Figures $3 a$ a) and 3c) and Figs. 4a) and 4c) show the details of sail-to-boom connections for the five-point and multiple-point connected sail designs, respectively. The model of the five-point connected sail had 5,613 nodes and 10,474 elements and the multiple-point connected sail had 5,613 nodes and 10,818 elements.

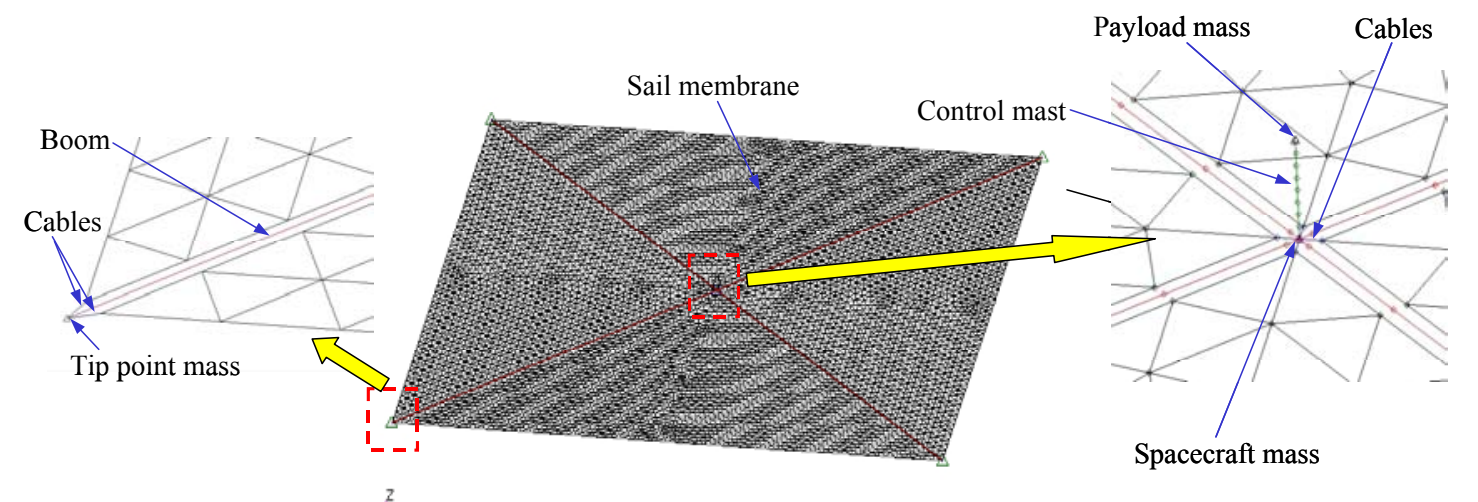

a) Corner sail region

b) Sail model

Figure 3: Five-point connected sail model.

c) Central sail region

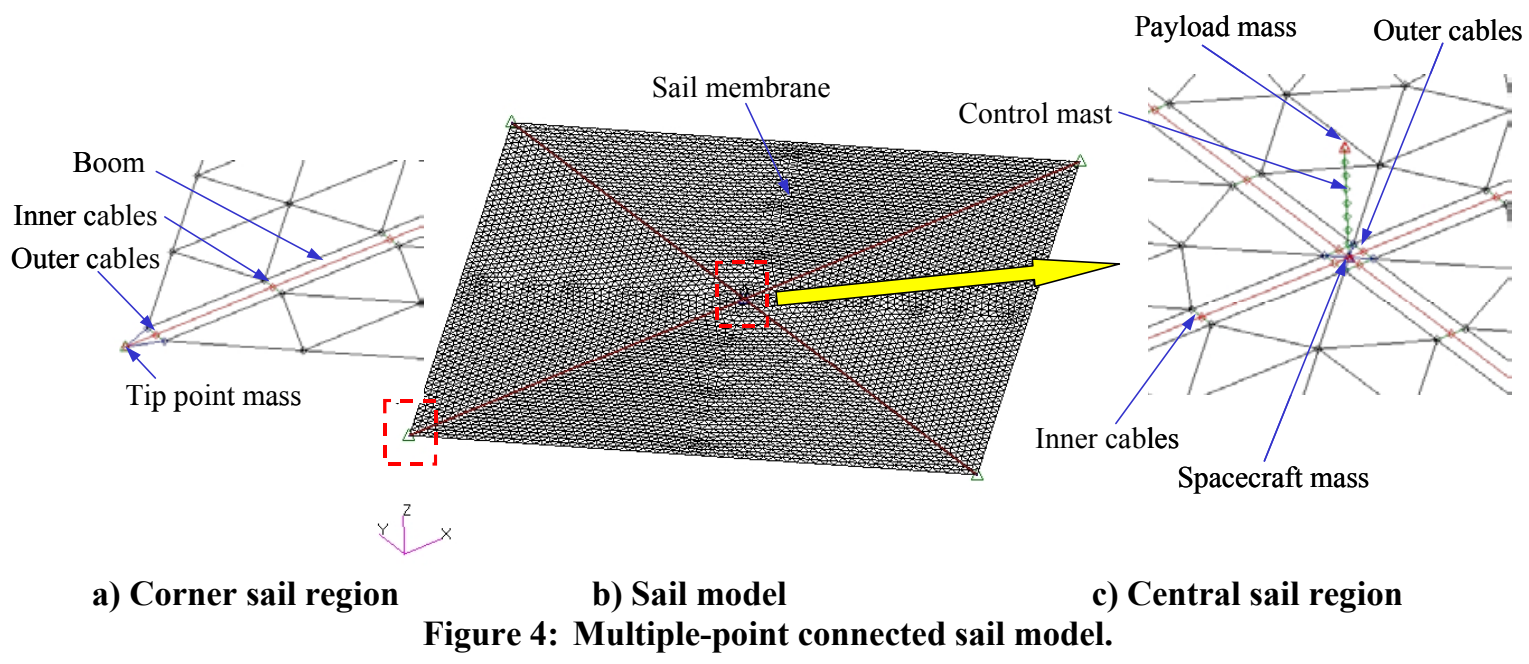

A fictitious thermal load simulated the pre-tensioning of the sail membrane by inducing contraction of the cables that connect the sail to the boom. Each node had a reference temperature value of $0^{\circ} \mathrm{C}$. A negative temperature was applied to all nodes in each model, and only those components with a non-zero positive coefficient of thermal expansion (CTE) would experience a contraction. All components of the solar sail were assigned a zero CTE, except for the cables. The cables shown in Figs. 3a) and 3c) for the five-point connected model were assigned a CTE of $4.6 \times 10^{-6} /{ }^{\circ} \mathrm{C}$. Thus, the sail pre-tensioning is applied at the vertices of each triangular sail region for the five-point connected solar sail. For the multiple-point connected solar sail, shown in Figs. 4a) and 4c), different values for the CTE were used for the cables. The inner cables, which are spaced every 1.6 meters along the length of the boom, were assigned a CTE of $4.6 \times 10^{-6} /{ }^{\circ} \mathrm{C}$. However, the outer cables, which connect the sail membrane to the booms at the vertices of each triangular sail region, were assigned a CTE of $4.6 \times 10^{-7} /{ }^{\circ} \mathrm{C}$. Thus, for the multiple-point 
connected design, the sail pre-tensioning is mainly applied on the sail edges parallel to the booms of each triangular sail region. Different pre-tensioning schemes were not explored.

Note that in Ref. 11, shell elements were used to model the sail, and beam elements were used to model both the booms and cables. However in the current study, models constructed with the three-node triangular S3 shell element and B31 beam elements experienced convergence problems during an analysis of the multiple-point connected solar sail. Attempts to achieve convergence by reducing the load step size and using the ABAQUS-specific stabilization feature were unsuccessful. Stabilization provides a mechanism for stabilizing unstable quasi-static problems through the addition of volume-proportional damping. This feature was used judiciously in the current study to achieve convergence when the standard quasi-static Newton-Raphson method failed. ABAQUS applies the volumeproportional damping to the model in the form of viscous forces added to the global equilibrium equations ${ }^{12}$. In order to achieve convergence in the nonlinear analyses, the shell elements were converted to membrane elements, and the beam elements modeling the cables were converted to truss elements upon the recommendation of ABAQUS Support. It was suggested that using the membrane and truss elements avoids element locking due to the extreme dimensions of the solar sails.

\section{A. Mesh Convergence Study}

Prior to initiating the parametric studies, a mesh convergence study was performed on the five-point connected $150 \mathrm{~m}$ solar sail design with three finite element models of increasing mesh fidelity. The $150 \mathrm{~m}$ solar sail was chosen for the mesh convergence study because it was expected to have the largest boom tip and sail membrane deflections based on the sail sizes used in the parametric studies. The details of each finite element model are listed in Table 2.

Table 2: Mesh convergence model summary.

\begin{tabular}{cccccc}
\hline \hline Model & Elements & Nodes & DOF's & $\Delta$ Temperature $\left({ }^{\circ} \mathbf{C}\right)$ & CPU Time (s) \\
\hline Model 1 & 2,682 & 1,529 & 9,194 & -489.9 & 17.6 \\
Model 2 & 10,474 & 5,613 & 33,678 & -533.0 & 58.7 \\
Model 3 & 20,758 & 10,917 & 65,502 & -567.2 & 182.3 \\
\hline \hline
\end{tabular}

The mesh convergence study focused on the maximum sail deflection and the vibration frequencies and mode shapes of a nonlinear equilibrium state including inertia relief. Inertia relief balances externally applied forces on a freeflying sail with inertial loads developed under steady-state rigid body acceleration. The reference point for the inertia relief was chosen to be at the center of mass of the solar sail, which was very close to the center of the solar sail. First, the sail membrane quadrants were pre-tensioned using a geometrically nonlinear analysis to $6,895 \mathrm{~N} / \mathrm{m}^{2}(1$ psi) at the center of each triangular sail membrane region as shown in Fig. 5 by applying a fictitious

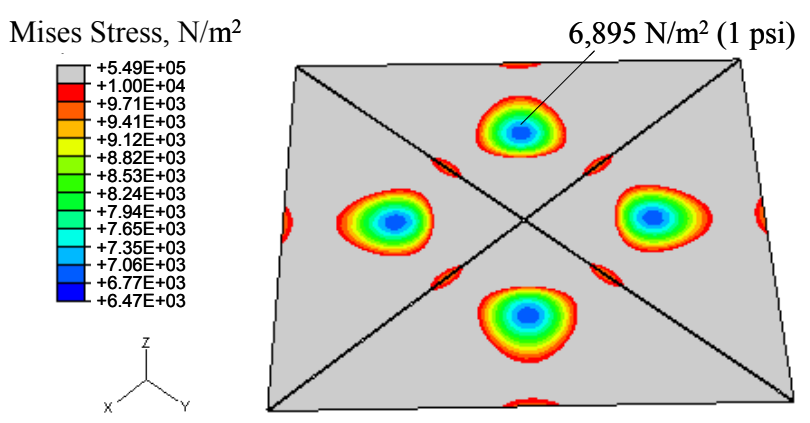

Figure 5: Mises stress distribution after applied pretension for five-point connected sail. thermal load to contract the cables. The baseline solution controls for each nonlinear analysis were a NewtonRaphson scheme with automatic load incrementation with a minimum load increment of $1 \times 10^{-6}$ times the applied loading and a maximum of 100 iterations per load increment. The pre-tension loads are induced by specifying a reduction in temperature from the reference state of the entire model as explained previously. To achieve a stress of $1.0 \mathrm{psi}$ in the center of each triangular sail region, different temperature changes were specified for each model, and these changes are given in Table 2. The initial temperature step applied $5 \%$ of the total temperature change.

Next, a solar pressure load of $9.12 \times 10^{-6} \mathrm{~N} / \mathrm{m}^{2}$ was applied in the negative $\mathrm{z}$-direction, normal to the pre-stressed sail membrane. This pressure value is representative of the anticipated solar flux at 1 astronomical unit (A.U.). Ten percent of the total pressure load was applied on the initial load step. In the final load case, the vibration frequencies and mode shapes about the nonlinear equilibrium state of the solar sail were computed using the default Lanczos eigensolver for Models 1 and 2. However, the Lanczos eigensolver with its default settings failed to extract the requested frequencies and vibration mode shapes for Model 3. A different eigensolver, the subspace iteration 
method, proved successful in determining reasonable vibration frequencies and mode shapes for Model 3. The CPU times on a Pentium IV $2.66 \mathrm{GHz}$ processor with $768 \mathrm{MB}$ of RAM are included in Table 2 for each model.

The maximum sail deflection, which occurs at the center of the outer edge of each triangular sail region, and the first five non-rigid-body frequencies of the three models are listed in Table 3. The reported maximum deflections are relative deflections to the center of the solar sail, because the inertia relief analysis includes rigid body translations and rotations about the center node of the model. The differences between the maximum sail deflection and the first five non-rigid-body frequencies of Models 1 and 2 were less than 1.6\%, while the differences between Models 2 and 3 were less than $0.6 \%$. As Model 2 appears to be sufficiently accurate and relatively efficient in terms of CPU time, its mesh was used throughout the remainder of the studies. A mesh convergence study was not performed on the multiple-point connected sail.

Table 3: Mesh convergence results summary.

\begin{tabular}{cccccc}
\hline \hline Model & $\begin{array}{c}\text { Maximum Sail } \\
\text { Deflection }(\mathbf{m})\end{array}$ & $\begin{array}{c}\mathbf{1}^{\text {st }} \text { Frequency } \\
(\mathbf{H z})\end{array}$ & $\begin{array}{c}\mathbf{2}^{\text {nd }} \text { Frequency } \\
(\mathbf{H z})\end{array}$ & $\begin{array}{c}\mathbf{3}^{\text {rd }}, \mathbf{4}^{\text {th }} \text { Frequency } \\
\mathbf{( H z )}\end{array}$ & $\begin{array}{c}\mathbf{5}^{\text {th }} \text { Frequency } \\
(\mathbf{H z})\end{array}$ \\
\hline Model 1 & -0.768 & 0.0104 & 0.0130 & 0.0162 & 0.0167 \\
Model 2 & -0.777 & 0.0104 & 0.0128 & 0.0160 & 0.0166 \\
Model 3 & -0.779 & 0.0104 & 0.0127 & 0.0159 & 0.0165 \\
\hline \hline
\end{tabular}

\section{B. Five-Point Connected Solar Sail}

The results from Model 2 of the mesh convergence study for the five-point connected sail model are presented below. A contour plot of the out-of-plane deflection superimposed on the deformed geometry (scaled $20 \times$ ) is shown in Fig. 6. The maximum relative sail deflection is at the center of the outer edge of each triangular sail region. The minimum principal stress distribution after the solar pressure load is applied is shown in Fig. 7. The regions in dark gray represent negative stresses where sail wrinkling may occur. Sail-wrinkling research has been an area of focus in past and current literature. There are two schemes implemented in Refs. 13, 14, and 15 that are implemented in an ABAQUS user subroutine to selectively adjust the material properties to eliminate negative stress regions in the analysis. Both methods predict the regions where wrinkles occur and their orientation; however, the tension field method $^{15}$ also employs an external algorithm to determine the amplitudes of wrinkles. Additional efforts by Tessler et al. ${ }^{16}$ include the prediction of wrinkled regions with their amplitudes and orientations using shell element models. The wrinkle deformations are induced through initial geometric imperfections in the mesh with peak amplitudes on the order of $10 \%$ of the shell thickness. The use of these schemes was beyond the scope of the current study but continue to be an active area of study ${ }^{17}$. The first five non-rigid-body mode shapes are shown in Fig. 8 for the fivepoint connected sail. The mode shapes were all sail-membrane dominated. The lowest mode shape resembles the "drum" mode shape reported in Ref. 10.

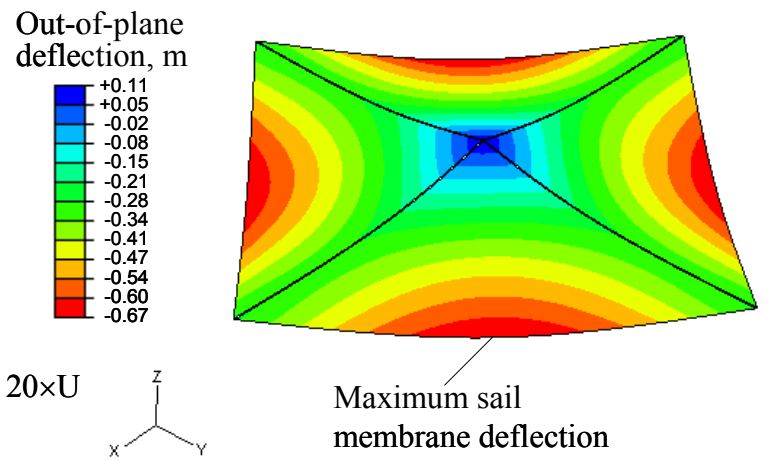

Figure 6: Out-of-plane deflection of five-point connected sail.

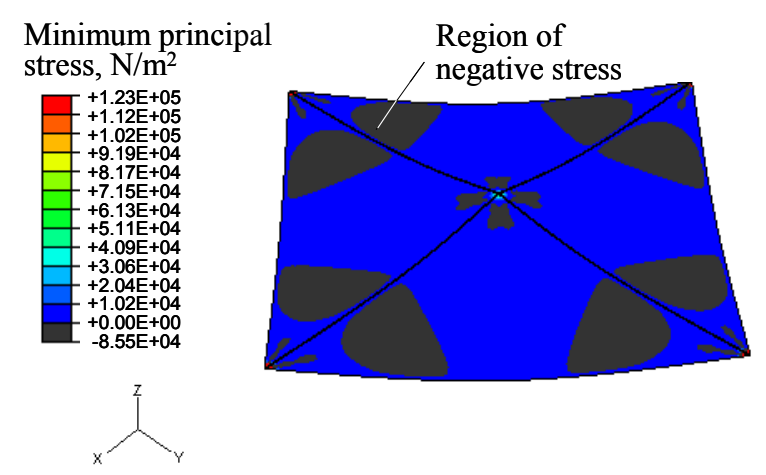

Figure 7: Minimum principal stress distribution of five-point connected sail. 


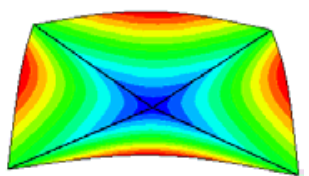

a) Mode 1 at $0.0105 \mathrm{~Hz}$

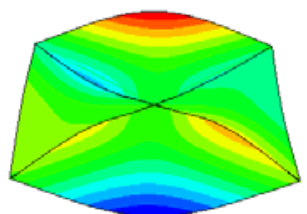

d) Mode 4 at $0.0158 \mathrm{~Hz}$

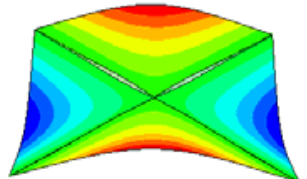

b) Mode 2 at $0.0127 \mathrm{~Hz}$

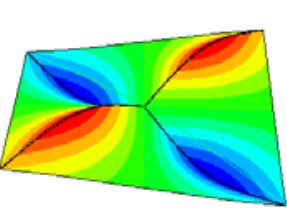

e) Mode 5 at $0.0165 \mathrm{~Hz}$

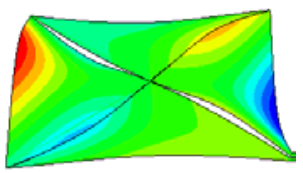

c) Mode 3 at $0.0158 \mathrm{~Hz}$

Out-of-plane

Deformation

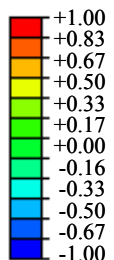

Figure 8: First five non-rigid-body mode shapes of five-point connected sail.

\section{Multiple-Point Connected Solar Sail}

The multiple-point connected sail model was analyzed with the same loading procedure as the fivepoint connected sail model, except that convergence was achieved when the stabilization parameter was set to *STATIC, STABILIZE $=2 \times 10^{-4}$ during the solar pressure load step. The CPU time on a Pentium IV 2.66 GHz processor was $1493.4 \mathrm{CPU}$ seconds. First, the sail membrane quadrants were pre-tensioned using a geometrically nonlinear analysis to $6,895 \mathrm{~N} / \mathrm{m}^{2}(1$ psi) at the center of each triangular sail membrane region as shown in Fig. 9 by applying a fictitious thermal load to contract the cables. The stress distribution after the pre-tension load is applied to the sail membrane is shown in Fig. 9. The stress

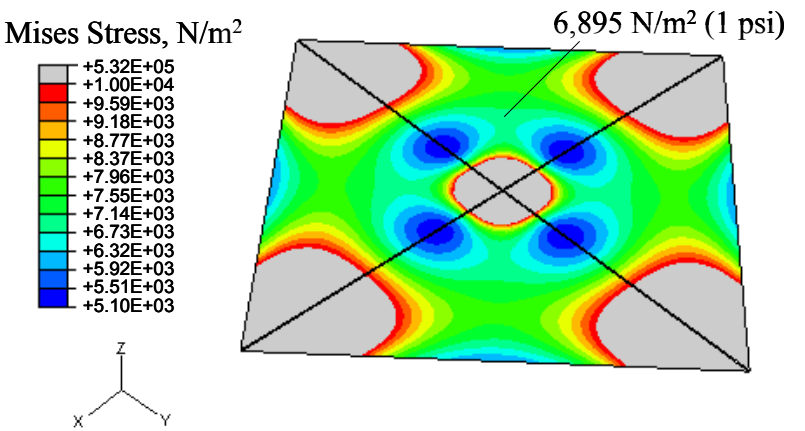

Figure 9: Mises stress distribution after applied pretension for multiple-point connected sail. distribution is different than that of the five-point connected sail because the pre-tensioning was mainly applied on the sail edges parallel to the booms. A study of different pre-tensioning schemes and their effect on the stress distribution over the sail membrane was considered beyond the scope of the current study. A contour plot of the out-of-plane deflection (scaled 20x) superimposed on the deformed geometry is shown in Fig. 10. The maximum sail deflection is again located at the center of the outer edge of each triangular sail region. However, the center of the solar sail along the booms has a lower deflection than the same region in the five-point connected sail. The minimum principal stress distribution after the solar pressure load is applied is shown in Fig. 11.

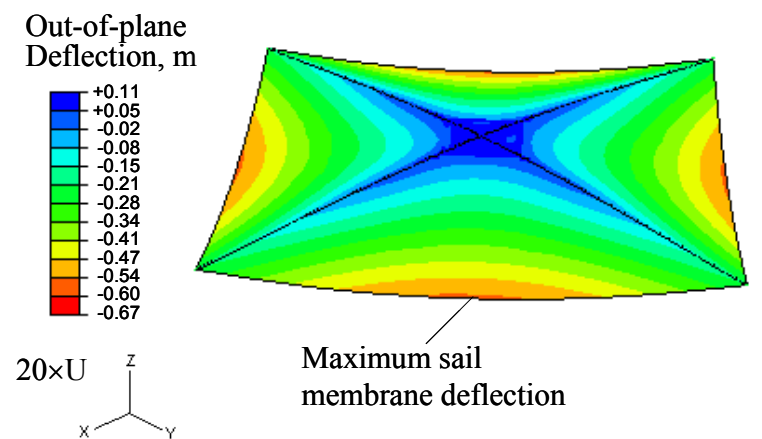

Figure 10: Out-of-plane deflection of multiplepoint connected sail.

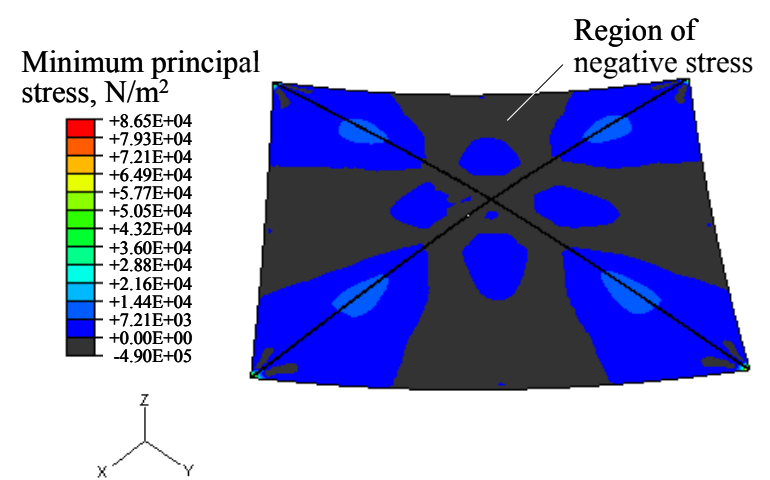

Figure 11: Minimum principal stress distribution of multiple-point connected sail. 
The sail membrane of the multiple-point connected sail has a different stress distribution than the five-point connected sail. The sail membrane of the multiple-point connected sail has a larger area with negative minimum principal stresses. This distribution may be attributed to the scheme by which the sail membrane was pre-tensioned. Other pre-tensioning schemes for the multiple-connected sail to reduce the regions of negative stress were not explored. The maximum sail deflection (relative to the center of the solar sail) and the first five non-rigid-body frequencies of the multiple-point connected sail are listed in Table 4. Again, the first five non-rigid-body mode shapes are sail-membrane dominated as shown in Fig. 12. The lowest mode shape is again the "drum" mode shape. However, local deformations appear in modes 2-5 in Figs. 12b)-12e), which are not believed to be physically meaningful. These local deformations are located in the same regions of the model that exhibited negative principal stresses in Fig. 11. Adler ${ }^{14}$ indicated that frequencies and mode shapes computed with membrane elements without considering sail membrane wrinkling could possess localized deformations that are artifacts of the negative minimum principal stresses in the sail membrane. Thus, only the first non-rigid-body frequency and corresponding mode shape are presented for the multiple-point connected models throughout the remainder of the studies described herein.

Table 4: Maximum sail deflection and frequencies of multiple-point connected model.

\begin{tabular}{cccccc}
\hline $\begin{array}{c}\text { Maximum Sail } \\
\text { Deflection }(\mathbf{m})\end{array}$ & $\begin{array}{c}\mathbf{1}^{\text {st }} \text { Frequency } \\
(\mathbf{H z})\end{array}$ & $\begin{array}{c}\mathbf{2}^{\text {nd }} \text { Frequency } \\
(\mathbf{H z})\end{array}$ & $\begin{array}{c}\mathbf{3}^{\text {rd }} \text { Frequency } \\
(\mathbf{H z})\end{array}$ & $\begin{array}{c}\mathbf{4}^{\text {th }} \text { Frequency } \\
(\mathbf{H z})\end{array}$ & $\begin{array}{c}\mathbf{5}^{\text {th }} \text { Frequency } \\
(\mathbf{H z})\end{array}$ \\
\hline-0.633 & 0.0123 & 0.0140 & 0.0143 & 0.0190 & 0.0191 \\
\hline \hline
\end{tabular}

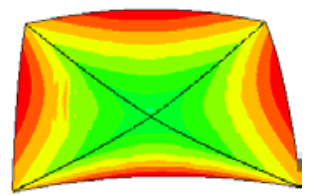

a) Mode 1 at $0.0123 \mathrm{~Hz}$

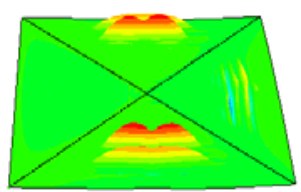

d) Mode 4 at $0.0190 \mathrm{~Hz}$

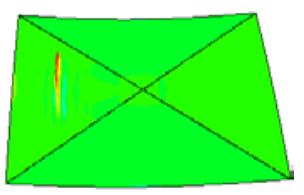

b) Mode 2 at $0.0140 \mathrm{~Hz}$

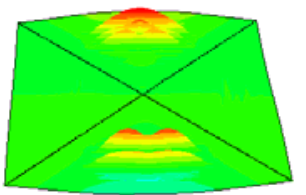

e) Mode 5 at $0.0191 \mathrm{~Hz}$

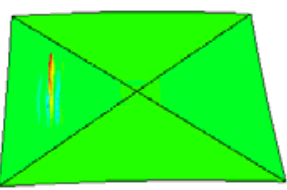

c) Mode 3 at $0.0143 \mathrm{~Hz}$

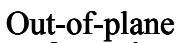

Deformation

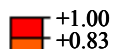

$\overbrace{X}^{Z} \begin{array}{r}+0.83 \\ +0.67 \\ +0.50 \\ +0.33\end{array}$

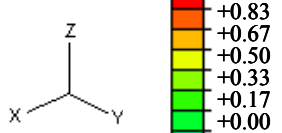

\begin{tabular}{|r|}
-0.16 \\
\hline+0.33 \\
\hline \\
-0.50 \\
-0.67
\end{tabular}

Figure 12: First five non-rigid-body mode shapes of multiple-point connected sail.

\section{Solar Sail Parametric Studies}

Parametric studies on the two square solar sail design concepts will be used to investigate the effects of sail size, sail membrane pre-stress, sail membrane thickness, and boom stiffness on the structural responses of the sail. Baseline parameters for the parametric studies are a $150 \mathrm{~m}$ solar sail with a $2.5 \mu \mathrm{m}$ sail membrane thickness pretensioned to $6,895 \mathrm{~N} / \mathrm{m}^{2}(1 \mathrm{psi})$ in the center of the sail membrane quadrant. The baseline thickness for the solar sail booms was $7.5 \mu \mathrm{m}$. All other properties and masses were assumed to be constant and were listed previously. Response quantities of interest include sail and boom deformations, boom compressive loads, and vibration frequencies.

\section{A. Boom Stiffness Studies}

Parametric studies were performed on the $150 \mathrm{~m}$ five-point connected design where the boom thickness was adjusted to affect a change in the boom stiffness for a constant diameter boom. The boom thickness was varied from $4.0 \mu \mathrm{m}$ to $41.0 \mu \mathrm{m}$, while all other material properties and dimensions were held constant. The largest thickness is the same value as in Ref 11. A parametric study was not performed on the multiple-point connected solar sail design, but is planned for future work. 
As the boom thickness was varied, the mass of the solar sail varied from $383.64 \mathrm{~kg}$ to $426.63 \mathrm{~kg}$, and the effective bending stiffness, EI, also varied from $37.2 \times 10^{3}$ to $382 \times 10^{3} \mathrm{~N}-\mathrm{m}^{2}$. We can define a factor of safety as the buckling load for a fixed-free boundary condition normalized by the maximum load in the boom after the sail solar pressure is applied. For the thinnest, or least stiff, boom where the boom thickness is $4.0 \mu \mathrm{m}$, the factor of safety as defined above was 3.2. For the baseline boom thickness of $7.5 \mu \mathrm{m}$, the factor of safety was 15 , and for the thickness boom with a thickness of $41.0 \mu \mathrm{m}$, the factor of safety was 83 . The thickest boom was used in the Ref. 11 study and is anticipated to be too stiff to be of interest. However, additional load conditions and analyses are required to establish whether a given boom's stiffness is sufficient to provide an acceptable safety margin.

A nonlinear analysis was performed including inertia relief, and the values selected for the boom thickness were 4.0, 4.5, 5.0, 6.0, 7.5, 10.0, 25.0 and $41.0 \mu \mathrm{m}$. The applied temperature change to pre-tension the sail was modified to achieve a $6,895 \mathrm{~N} / \mathrm{m}^{2}$ stress in the center of each triangular sail region for each boom thickness value. A successful solution is achieved and frequencies and mode shapes are calculated with the ABAQUS solution inputs described in the Mesh Convergence Study section when the boom thickness is above $4.5 \mu \mathrm{m}$. When the boom thickness was $4.5 \mu \mathrm{m}$, however, the frequencies and mode shapes were not calculated with the Lanczos eigensolver until the maximum number of iterations was increased from its default value to 100 and a shift point of $0.0001 \mathrm{~Hz}^{2}$ was specified. The Lanczos solver required additional computational guidance and more time to distinguish amongst the rigid and non-rigid-body frequencies and mode shapes during an analysis including inertia relief. When the boundary conditions were changed from a free-flight to a fixed central node boundary condition, the frequencies and mode shapes were successfully generated with the default Lanczos eigensolver inputs. As the boom thickness was further reduced to $4.0 \mu \mathrm{m}$, the analysis exhibited convergence difficulties as the solar pressure load was applied using the solution controls listed in the Mesh Convergence Study. Convergence was achieved when the stabilization parameter was set to *STATIC, STABILIZE $=1 \times 10^{-8}$ during the solar pressure load step. The frequencies and mode shapes were successfully extracted using the modified Lanczos solutions controls listed above.

The results indicate that the boom thickness has a strong impact on the static response of the solar sail. The maximum sail and boom tip deflections are shown in Fig. 13 as a function of the boom thickness. Both the boom and sail deflections increase rapidly as the boom thickness is reduced below the baseline value of $7.5 \mu \mathrm{m}$. The boom compression loads after applying the sail pre-tensioning and solar pressure loads are shown in Fig. 14 as a function of the boom thickness. The plots indicate that the boom loads are smaller after the sail pre-tensioning than after the solar pressure load. As the boom thickness is decreased, the difference in the boom loads is reduced.

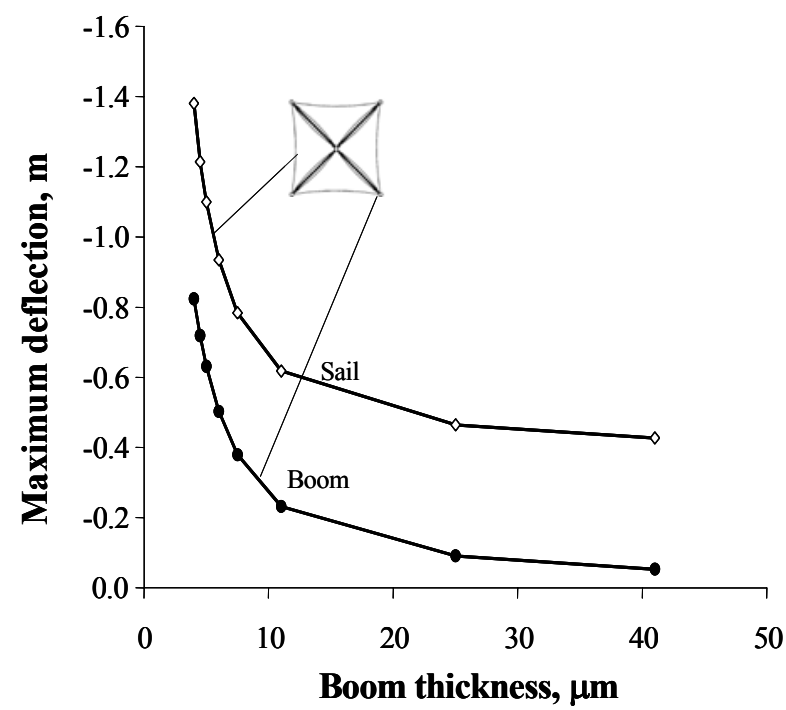

Figure 13: Sail and boom tip deflections for boom thickness study.

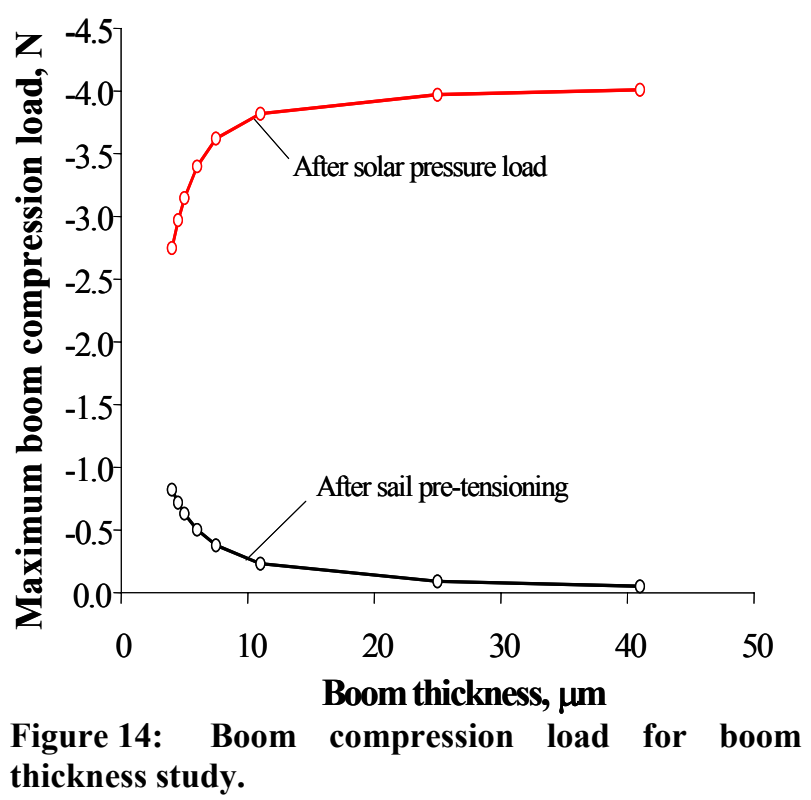

thickness study.

Not surprisingly, the results also indicate that the boom stiffness has a strong impact on the dynamic response of the solar sail. The first five non-rigid body frequencies are shown in Fig. 15 as a function of boom thickness. The frequencies and mode shapes are identified by their order from the analysis of the baseline solar sail model with a thickness of $7.5 \mu \mathrm{m}$ shown in Fig. 8. Note that the third and fourth frequencies are coincident. The mode shapes associated with the first four frequencies are invariant as the boom thickness is varied from 4.0 to $41.0 \mu \mathrm{m}$. 
However, the fifth mode shape of the baseline solar sail model (See Fig. 8e)) is associated with higher frequencies as the boom thickness is varied. For example, this mode shape is associated with the seventh, eighth, and ninth frequencies for boom thicknesses of 10.0, 41.0, and 25.0 $\mu \mathrm{m}$, respectively. Three regions are seen in the figure where the frequencies coalesce or nearly coalesce: at boom thicknesses below $5.0 \mu \mathrm{m}$, at $10.0 \mu \mathrm{m}$, and at 41.0 $\mu \mathrm{m}$. Regions where frequencies coalesce and where modes may also cross or veer are of interest dynamically since the 1:1 frequency relationship can enable nonlinear coupling of the underlying vibration modes in the presence of disturbances. Note that other commensurate frequency relationships, e.g. 1:2, can also enable modal coupling. Mode crossing is observed as the fifth vibration frequency (and corresponding mode shape) crosses the third/fourth frequency (and mode shape) as the boom thickness is decreased below $5.0 \mu \mathrm{m}$. Mode veering also is observed as the second frequency (and mode shape) veers from a near 1:1 relationship with the first frequency

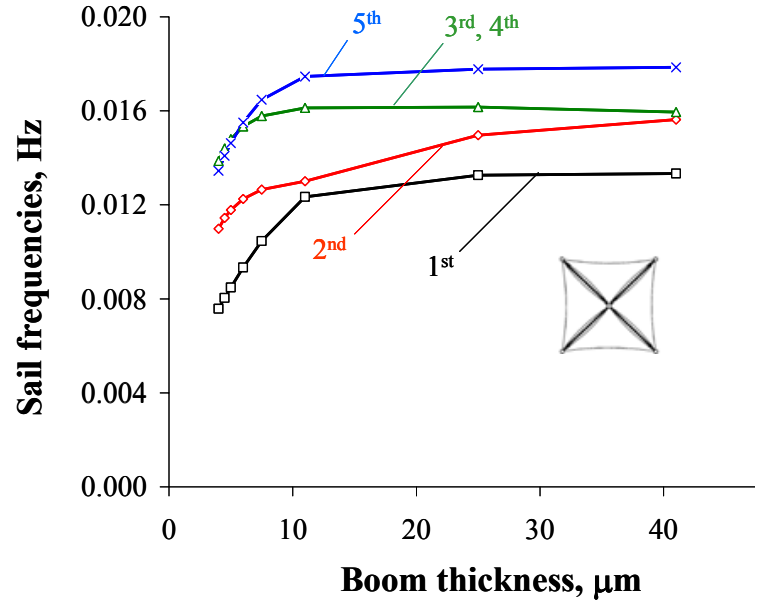

Figure 15: Sail frequencies for boom thickness study. at $10.0 \mu \mathrm{m}$ to a near $1: 1$ relationship with the third/fourth frequency (and mode shape) at $41.0 \mu \mathrm{m}$. Also note that the region of mode crossing below a boom thickness of 5.0 $\mu \mathrm{m}$ is where the solution controls required adjustments to obtain solutions, which is indicative of the computational challenges manifested by the solar sail geometric parameters. A study of the dynamic response of the solar sail designs to investigate modal coupling in the presence of disturbances was beyond the scope of the current work.

\section{B. Sail Size Studies}

Parametric studies were performed on both square solar sail designs by scaling the in-plane dimensions of the $150 \mathrm{~m}$ finite element models to achieve sail sizes from 20 to $150 \mathrm{~m}$ per edge. The length of the control mast was kept constant at $2 \mathrm{~m}$ in length for all analyses, as were all material properties and concentrated masses, including the spacecraft and payload masses. As the sail size was reduced, the mass of the solar sail also decreased. The mass of the solar sails varied from $295 \mathrm{~kg}$ for the $20 \mathrm{~m}$ solar sail to $388 \mathrm{~kg}$ for the $150 \mathrm{~m}$ solar sail.

Nonlinear analyses were performed using inertia relief for both sail designs for sail sizes of 20, 40, 70, 100, 125, and $150 \mathrm{~m}$ per edge. The applied temperature change to the cables for pre-tensioning the sail was modified to achieve a $6,895 \mathrm{~N} / \mathrm{m}^{2}$ stress level at the center of each triangular sail membrane region. The stabilization feature in ABAQUS was required to achieve convergence with the multiple-point connected models. Whenever the stabilize parameter was used, the smallest stabilization value that achieved convergence was specified. This value ranged from a minimum of $2 \times 10^{-4}$ for the 125 and $150 \mathrm{~m}$ sails to a maximum of $3 \times 10^{-4}$ for the $20,40,70$, and 100 m sails.

The maximum sail and boom tip deflection are shown in Fig. 16 as a function of sail size. As the sail size is increased for each sail design, both the sail and boom tip deflections increase nonlinearly as expected. The analyses predicted that the sail and boom tip deflections for the fivepoint connected sail design (black curves in figure) are larger than the multiple connected sail design (red curves in figure). The maximum boom compressive loads for both sail designs also were computed (not shown) and the boom loads increased linearly as the sail size was increased. The boom compressive loads of the multiple-point connected solar sail were slightly lower than the five-point connected

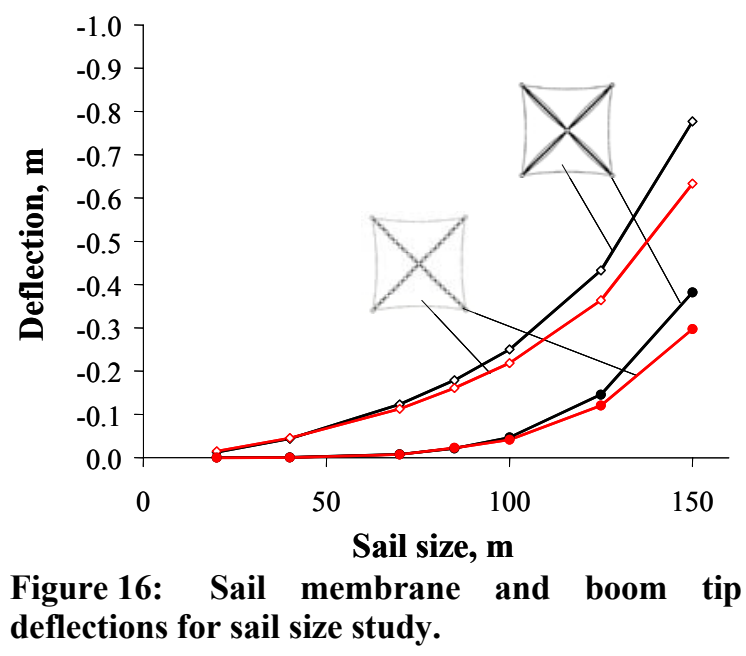
sail design. 
The first five non-rigid-body frequencies are shown in Fig. 17 as a function of sail size for the five-point connected sail. The results indicate that the first four frequencies separate as the sail size is increased; however, they are closely spaced for all sail sizes. The frequencies decrease as the sail size is increased. The frequencies and mode shapes are identified by their order from the analysis of the baseline solar sail model of $150 \mathrm{~m}$ shown in Fig. 8. For all sail sizes, the third and fourth frequencies are coincident and the first four mode shapes are similar. However, the fifth mode shape of the baseline solar sail model (See Fig. 8e)) is associated with higher frequencies as the sail size is decreased. For example, this mode shape is associated with the seventh frequency of the $125 \mathrm{~m}$ sail and the ninth frequency for sail sizes of $100 \mathrm{~m}$ or less. Only the first vibration frequency and mode shape was determined for the multi-point connected solar sail as discussed previously. A comparison of the first non-rigid-body frequency for both sail designs is shown in Fig. 18. The results indicate that the first sail frequencies in both sail designs are very close to each other. The frequency of the five-point connected sail is slightly lower for sail sizes of $40 \mathrm{~m}$ and larger.

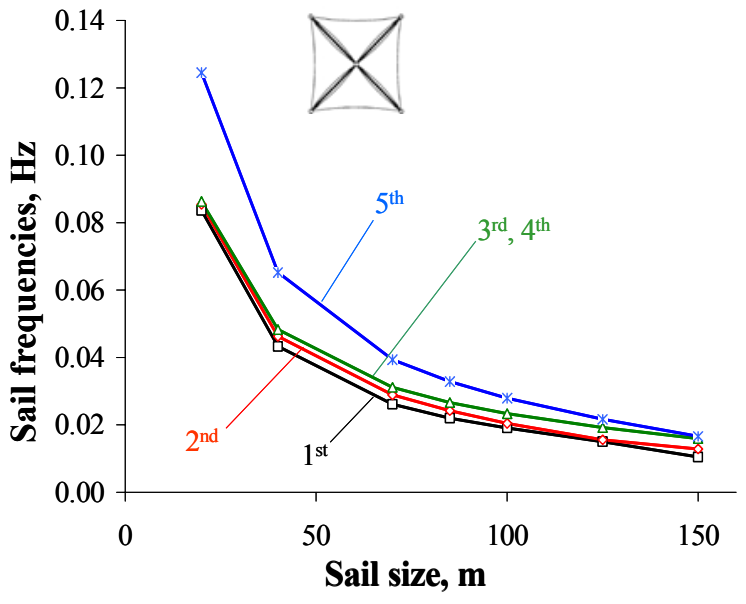

Figure 17: Frequencies of five-point connected sail for sail size study.

\section{Sail Membrane Thickness Studies}

Parametric studies were performed on both solar sail designs by varying the sail membrane thickness from 0.5 to $10 \mu \mathrm{m}$. All other properties, dimensions, and masses were kept constant. As the sail membrane thickness is increased, the mass of the solar sail also increased, ranging from $318 \mathrm{~kg}$ for a sail membrane thickness of $0.5 \mu \mathrm{m}$ to $648 \mathrm{~kg}$ for a sail membrane thickness of 10 $\mu \mathrm{m}$.

Again, the applied temperature to the cables for pretensioning the sail had to be modified for each analysis to obtain the $6,895 \mathrm{~N} / \mathrm{m}^{2}$ stress level in the center of each triangular sail membrane region. The stabilization feature in ABAQUS was used to achieve convergence for the multiple-point connected models. This value ranged from a minimum value of $2 \times 10^{-4}$ for the $2.5 \mu \mathrm{m}$ sail membrane thickness model to a maximum value of $8 \times 10^{-4}$ for the $0.5 \mu \mathrm{m}$ sail membrane thickness model.

The maximum sail and boom tip deflection is shown in Fig. 19 as a function of sail membrane thickness. Again, the analyses predicted that the sail and boom tip deflections were larger in the five-point connected sail

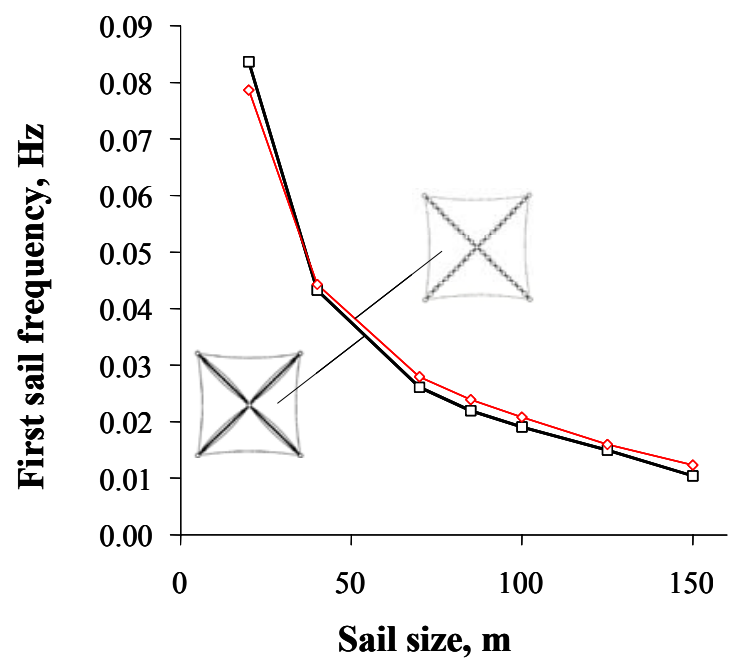

Figure 18: Comparison of first sail frequency of both sail designs for sail size study.

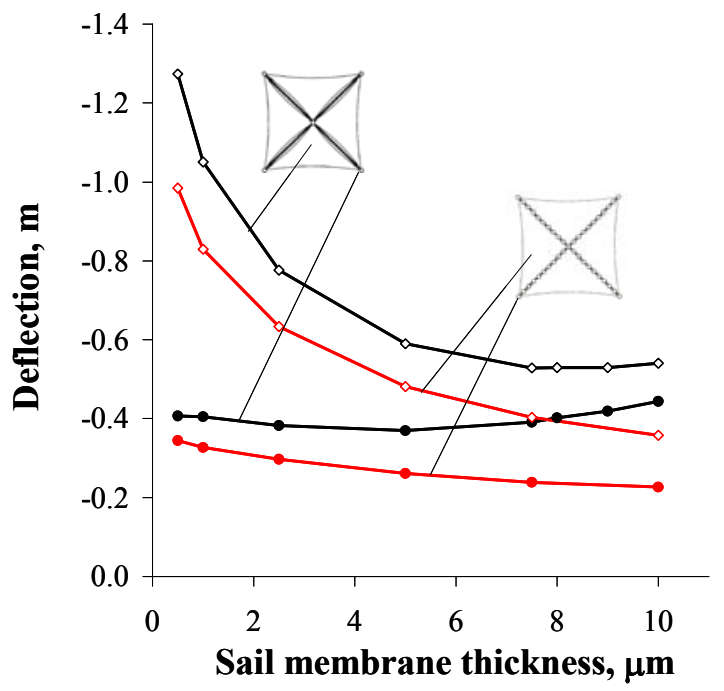

Figure 19: Sail membrane and boom tip deflections for sail membrane thickness study. 
design. However, in the five-point connected sail design, the sail and boom tip deflections initially decrease as the sail membrane thickness is increased, but then increase as the sail membrane thickness is increased from 7.5 to 10 $\mu \mathrm{m}$. This behavior may be attributable to inertia relief. As the sail membrane thickness is increased, the mass of the solar sail also increases, and therefore a larger acceleration load is balanced at the inertia relief reference point.

The maximum boom compressive loads for both sail designs also were computed, and the boom loads increased linearly as the sail sizes increased. The boom compressive loads of the multiple-point connected solar sail were slightly lower than the five-point connected design.

The first five non-rigid-body frequencies are shown in Fig. 20 as a function of sail size for the five-point connected sail design. The results indicate that the frequencies decrease as the sail size is increased. For all sail membrane thicknesses, the third and fourth vibration frequencies are repeated. The frequencies and mode shapes are identified by their order from the analysis of the baseline solar sail model with a sail membrane thickness of $2.5 \mu \mathrm{m}$ shown in Fig 8. For membrane thicknesses above $2.5 \mu \mathrm{m}$, the fifth frequency crosses the third and fourth mode frequencies. Again, the second vibration frequency veers from being near the first frequency to the fifth frequency as the thickness is varied from $1 \mu \mathrm{m}$ to $10 \mu \mathrm{m}$. Again, only the first vibration frequency and mode shape were reported for the multiple-point connected design.

A comparison of the first non-rigid-body frequency for both sail designs is shown in Fig. 21. The results indicate that the first sail frequency is lower for the five-point connected sail design. However, as the sail membrane thickness is increased, the first sail frequencies of the two designs approach each other.

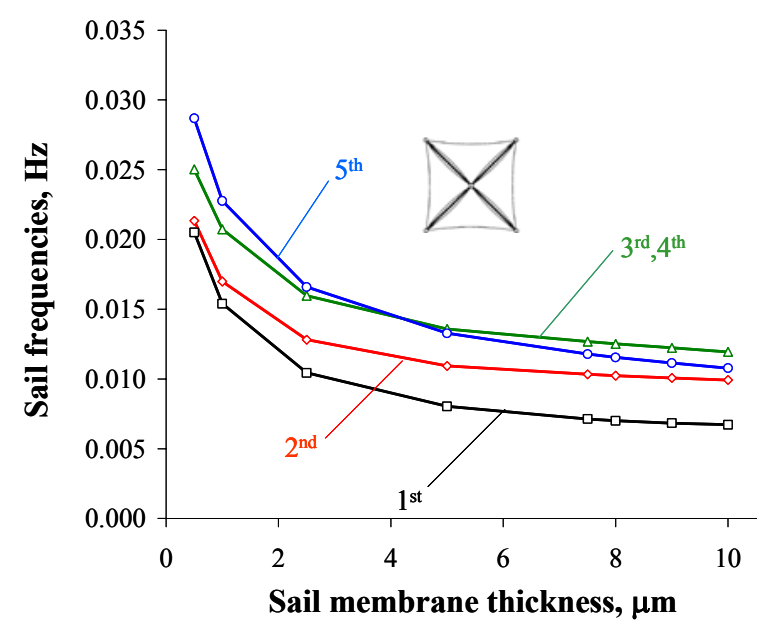

Figure 20: Frequencies of five-point connected sail for sail membrane thickness study.

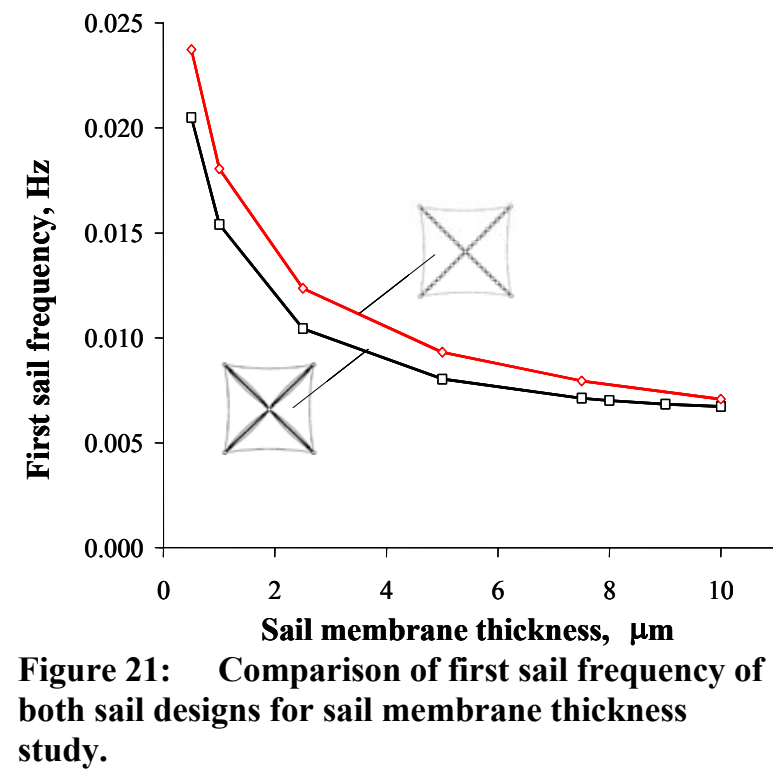

study.

\section{Sail Membrane Pre-stress Studies}

Parametric studies were performed on the $150 \mathrm{~m}$ five-point connected design where the sail pre-stress level at the center of each triangular sail membrane was varied from 0.1 to 5 psi. All other properties, dimensions, and masses were kept constant. A parametric study was not performed on the multiple-point connected solar sail design, but is planned for future work.

A nonlinear analysis was performed including inertia relief as the sail membrane was pre-stressed to values of $0.1,1,2,3$, and 5 psi. The temperatures applied to the cables were modified to achieve the desired pre-stress level at the center of each triangular sail membrane. The maximum number of iterations for the Lanczos eigensolver was increased from a default value of 20 to 100 , and a shift point of $0.0001 \mathrm{~Hz}^{2}$ was specified for all pre-stress levels other than $1 \mathrm{psi}$. These solution parameters were specified a priori based on lessons learned during the boom thickness study. No other adjustments in the solution control parameters were made.

The results indicate that the sail pre-stress level has a strong impact on the response of the solar sail. The maximum sail and boom tip deflections are shown in Fig. 22 as a function of the pre-stress level. The sail deflections indicate an inflection point as the pre-stress level increases from 1 to $3 \mathrm{psi}$, while the boom deflections continue to increase and approach the sail deflection as the pre-stress reaches 5 psi. The boom compression levels increased linearly as the pre-stress levels increased (not shown). The first five non-rigid-body frequencies are shown 
in Fig. 23 as a function of the sail pre-stress level. The frequencies and mode shapes are identified by their order from the analysis of the baseline solar sail model pre-stressed to 1 psi shown in Fig. 8 . At a pre-stress of 1.0 psi, the third and fourth frequencies are repeated. As the pre-stress level is increased to $5 \mathrm{psi}$, the third and fourth vibration frequencies cross the fifth vibration frequency, and the second frequency value begins to veer toward the value of the third and fourth frequencies. For membrane pre-stress levels above $1.0 \mathrm{psi}$, the fifth frequency crosses the third and fourth frequencies. The first five mode shapes are similar for all pre-stress levels studied.

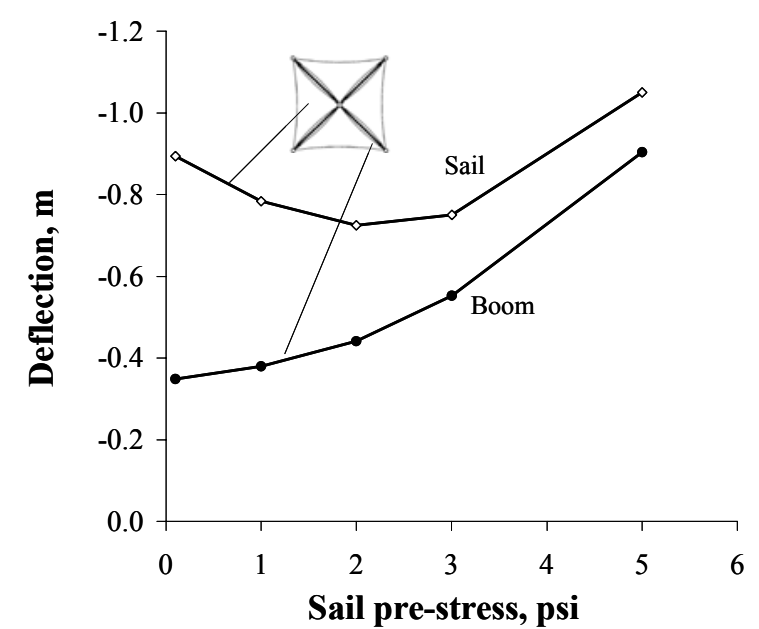

Figure 22: Sail and boom tip deflections for sail pre-stress study.

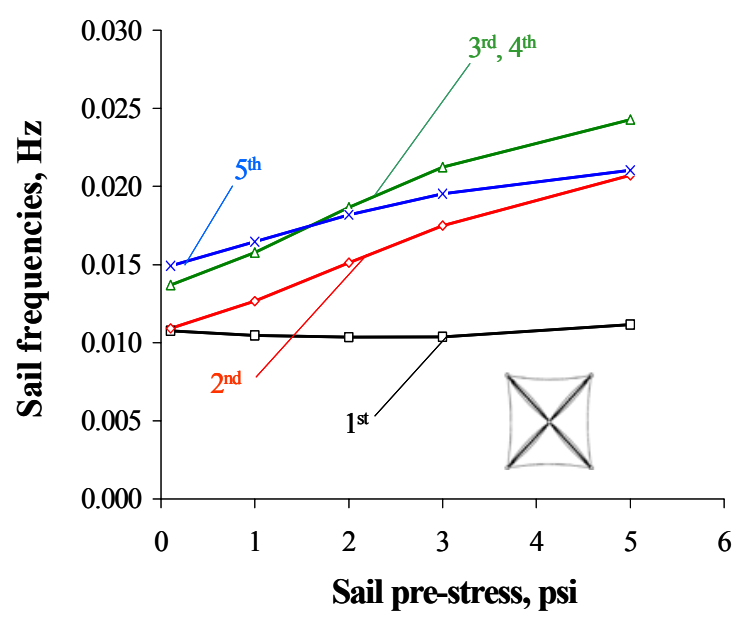

Figure 23: Sail frequencies for sail pre-stress study.

\section{Concluding Remarks}

Parametric studies using high fidelity computational methods and models were performed on two generic system-level solar sail design concepts. Computational difficulties encountered during the analyses were identified and strategies to mitigate them also were identified. The strategies included the use of the stabilization feature within the ABAQUS finite element software to achieve convergence in the nonlinear analyses and the use of modified eigensolver inputs to determine the vibration frequencies and mode shapes. The analyses included geometric nonlinearity and inertia relief to simulate a free-flying sail, and used a Newton-Raphson scheme to apply sail pretensioning and solar pressure. The purpose of this paper was not to compare the benefits of one sail design over the other. Instead, the parametric studies described in this paper may be used to identify the general response trends associated with key parameters in the design of solar sails, and areas of potential nonlinear structural interactions for future studies.

Parametric studies were performed on two solar sail design concepts to investigate the effects of sail size, sail membrane pre-stress, sail membrane thickness, and boom stiffness on the sail membrane and boom deformations, boom compressive load, and vibration frequencies and mode shapes. One solar sail concept had four separate triangular sails attached to the booms by cables at five points, while the other had four separate triangular sails attached to the booms by cables at multiple attachment points along the length of the booms. Over the range of parameters studied, all parameters were shown to have a significant nonlinear effect on the sail membrane and boom tip deflections. It was found that the multiple-point connected sail had smaller sail and boom deformations in comparison to the five-point connected sail for a given design. For both solar sail concepts, the first five non-rigidbody vibration frequencies and modes were closely spaced and below $0.15 \mathrm{~Hz}$. Regions of mode crossing and/or mode veering as well as repeated or nearly coincident mode shapes were identified for nearly all parameters. These phenomena indicated regions of potential nonlinear modal interaction. Only the lowest non-rigid-body vibration frequency and mode shape were presented for the multiple-point connected sail design. For mode shapes above the lowest frequency, local deformation patterns that dominate the response were identified. These localized patterns were attributed to the presence of negative stresses in the sail membrane that were artifacts of the assumption of ignoring the effects of wrinkling in the modeling process, and were not believed to be physically meaningful. 


\section{Acknowledgments}

The authors acknowledge contributions to this paper in the area of modal coupling made by Dr. Suzanne Weaver Smith of the Department of Mechanical Engineering, University of Kentucky.

\section{References}

${ }^{1}$ Salama, M., McInnes, C. R., and Mulligan, P., "Gossamer Spacecraft Technology," Gossamer Spacecraft: Membrane and Inflatable Structures Technology for Space Applications, Edited by C.H.M. Jenkins, Progress in Astronautics and Aeronautics, Vol. 191, AIAA, Reston, VA, 2001.

${ }^{2}$ Wright, J., Space Sailing, Taylor and Francis Publishers, 1992.

${ }^{3}$ McInnes, C. R., Solar Sailing: Technology, Dynamics, and Mission Applications, Springer-Praxis Series in Space Science and Technology, 1999.

${ }^{4}$ Chmielewski, A. B., "Advanced Concepts," Gossamer Spacecraft: Membrane and Inflatable Structures Technology for Space Applications, Edited by C.H.M. Jenkins, Progress in Astronautics and Aeronautics, Vol. 191, AIAA, Reston, VA, 2001.

${ }^{5}$ Internet reference: http://www.teamencounter.com [cited 12 March 2004].

${ }^{6}$ Internet reference: http://solarsails.jpl.nasa.gov [cited 12 March 2004].

${ }^{7}$ West, J. L. and Derbes, B., "Solar Sail Vehicle System Design for the Geostorm Warning Mission," $41^{\text {st }}$ AIAA structures, Structural Dynamics and Materials Conference and Adaptive Structures Forum, AIAA-2000-5326, 2000.

${ }^{8}$ Genta, G., Brusa, E., C. Delprete, "Second approximation structural assessment of the Aurora solar sail spacecraft," Acta Astronautica, 54, pp. 115-125, 2003.

${ }^{9}$ Greschik, G. and Mikulas, M. M., "Design Study of a Square Solar Sail Architecture," $42^{\text {nd }}$ AIAA/ ASME/ASCE/AHS/ASC Structures, Structural Dynamics, and Materials Conference and Exhibit, Seattle, WA, AIAA-2001-1259, 2001.

${ }^{10}$ Murphy, D. M., Murphey, T. W., and Gierow, P. A., "Scalable Solar Sail Subsystem Design Considerations," 43 ${ }^{\text {rd }}$ AIAA/ASME/ASCE/AHS/ASC Structures, Structural Dynamics, and Materials Conference and Exhibit, Denver, CO, AIAA-20021703, 2002.

${ }^{11}$ Taleghani, B. K., Sleight, D. W., Muheim, D. M., Belvin, W. K., and Wang, J. T., “Assessment of Analysis Approaches for Solar Sail Structural Response," 39th AIAA/ASME/SAE/ASEE Joint Propulsion Conference and Exhibit, Huntsville, AL, AIAA2003-4796, 2003.

${ }^{12}$ ABAQUS/Standard User's Manual, Version 6.4.1, Hibbitt, Karlsson, and Sorensen, Inc., Pawtucket, RI, 2003.

${ }^{13}$ Adler, A. L., Mikulas, M. M., and Hedgepeth, J. M, "Static and Dynamic Analysis of Partially Wrinkled Membrane Structures," 41st AIAA/ASME/ASCE/AHS/ASC Structures, Structures Dynamics, and Material Conference, Atlanta, GA, AIAA2000-1810, 2000.

${ }^{14}$ Adler, A. L., "Finite Element Approaches for Static and Dynamic Analysis of Partially Wrinkled Membrane Structures," Ph.D. Dissertation, Aerospace Engineering Department, University of Colorado, Boulder, CO, December 2000.

${ }^{15}$ Liu, X., Jenkins, C.H., and Schur, W.W., "Fine Scale Analysis of Wrinkled Membranes," International Journal of. Computational Engineering Science, Vol. 1, No. 2, pp. 281-298, 2000.

${ }^{16}$ Tessler, A., Sleight, D. W., and Wang, J. T., "Nonlinear Shell Modeling of Thin Membranes with Emphasis on Structural Wrinkling," 44th AIAA/ASME/ASCE/AHS/ASC Structures, Structural Dynamics, and Materials Conference and Exhibit, Norfolk, VA, AIAA-2003-1931, 2003.

${ }^{17}$ Tessler, A., Sleight, D. W., "Toward Effective Shell Modeling Of Wrinkled Thin-Film Membranes Exhibiting Stress Concentrations," 45th AIAA/ASME/ASCE/AHS/ASC Structures, Structural Dynamics, and Materials Conference and Exhibit, Palm Springs, CA, AIAA-2004-1739, 2004. 